EPJ manuscript No.

(will be inserted by the editor)

\title{
The Phase Behavior of Mixed Lipid Membranes in Presence of the Rippled Phase
}

\author{
N. Shimokawa ${ }^{1}$, S. Komura ${ }^{1}$ and D. Andelman ${ }^{2}$ \\ 1 Department of Chemistry, Graduate School of Science and Engineering, Tokyo Metropolitan University, Tokyo 192-0397, \\ Japan \\ 2 School of Physics and Astronomy, Raymond and Beverly Sackler Faculty of Exact Sciences, Tel Aviv University, Ramat Aviv \\ 69978, Tel Aviv, Israel
}

Received: date / Revised version: date

\begin{abstract}
We propose a model describing liquid-solid phase coexistence in mixed lipid membranes by including explicitly the occurrence of a rippled phase. For a single component membrane, we employ a previous model in which the membrane thickness is used as an order parameter. As function of temperature, this model properly accounts for the phase behavior of the three possible membrane phases: solid, liquid and the rippled phase. Our primary aim is to explore extensions of this model to binary lipid mixtures by considering the composition dependence of important model parameters. The obtained phase diagrams show various liquid, solid and rippled phase coexistence regions, and are in quantitative agreement with the experimental ones for some specific lipid mixtures.
\end{abstract}

PACS. 87.16.Dg Membranes, bilayers, and vesicles. $-64.75 .+\mathrm{g}$ Solubility, segregation, and mixing

\section{Introduction}

In recent years, domain formation in biomembranes and artificial membranes has attracted great attention in connection to the so-called "raft formation" in biological cell membranes [1. It is believed that the appearance of such domains (rafts) in membranes plays an important role for various cell functions [2,3]. Beside their interest in biocellular processes, raft formation has a fundamental physical interest, because it offers an special example of two' dimensional phase separations coupled to internal and external membrane degrees of freedoms 4 . Using fluorescence microscopy or X-ray diffraction techniques, the lateral phase separation between different liquid phases, or between the solid and liquid phases has been observed for ternary-component vesicles consisting of saturated lipid, unsaturated lipid, and cholesterol [5, 6, 7, 8, 9]. Although the biological significance of rafts is not yet fully understood, it serves as our primary motivation to explore, from a physical point of view, phase transitions, domains and phase coexistence in multi-component lipid membranes.

Lateral phase separation in membranes occurs even for the simpler case of binary lipid mixtures without any added cholesterol. The coexistence is between liquid-like $\left(L_{\alpha}\right)$ and solid-like ( $L_{\beta^{\prime}}$ called "gel") phases, and it can be visualized using techniques such as two-photon fluorescence microscopy [10]. For example, for DPPC/DPPE lipid mixture, the solid domains exhibit morphologies such as hexagonal, dumbbell or dendritic shapes [10], while for DPPC/DLPC lipid vesicle the solid domains appear to be stripe-like and are monodispersed in their domain width [11. Shapes of solid domains found on spherical fluid vesicles has been also addressed theoretically [12]. By assuming the additivity of stretching and line energies, the model predicts a phase diagram including cap, ring and ribbon phase domains on a spherical surface.

Although visualization of domains embedded in fluid membranes has become possible only recently, the phase diagram of various binary lipid mixtures in bilayer membranes has been known for some time [13,14]. In a previous model investigated by some of us [15, 16], a coupling between composition and internal membrane structure was proposed and the resulting phase diagram was calculated. It includes a coexistence region between $L_{\alpha}$ and $L_{\beta^{\prime}}$ phases in agreement with experiments on specific lipid mixtures. However, for some lipids, such as DMPC or DPPC, another distinct solid-like phase is known to occur and is called the "rippled" or $P_{\beta^{\prime}}$ phase. The unique feature of this phase is that the membrane shape is spatially modulated while the lipid hydrocarbon tails are ordered. The specific phase diagrams of DMPC/DPPS, DPPC/DPPS [17, or DPPC/DPPE [18] mixtures have been explored in experiments, and show the three phases $\left(L_{\alpha}, L_{\beta^{\prime}}, P_{\beta^{\prime}}\right)$ together with the coexistence regions between them. However, these phase diagrams have not yet been considered theoretically.

The main objective of the present paper is to provide a simple model that describes the liquid-solid coexistence in binary lipid mixtures while considering the possibility 
N. Shimokawa, S. Komura, D. Andelman: The Phase Behavior of Mixed Lipid Membranes

of an intermediate rippled phase. Our starting point is a model introduced by Goldstein and Leibler (GL) that accounts for the succession of phase transitions as applied to single-component lipid membrane [19,20]. In the GL model, the membrane thickness is used as a scalar order parameter. We combined some of the ideas presented in the GL model with our own previous model for binary (or even ternary) lipid mixtures 15, 16. For binary lipid mixtures, the model parameters are taken to be dependent on the relative lipid composition, and resulting in several types of phase diagrams that are in quantitative agreement with experiments on specific lipid mixtures.

In the next section, we first review the GL model describing the phase transitions in a single-component lipid membrane. The corresponding mean-field phase diagram is obtained in terms of the temperature and membrane elastic constants. In Sec. 3, we propose an extension of the GL model for a two-component lipid membrane, and calculate the binary phase diagrams. Some discussions and comparison to other models are discussed in Sec. 4.

\section{Single-component lipid system}

In a single-component lipid bilayer, one typically observes a discontinuous first-order phase transition from the $L_{\alpha}$ to the $L_{\beta^{\prime}}$ phase as the temperature is decreased. This is called the "main transition" and is associated with ordering of the hydrocarbon tails of the lipid molecules. In the $L_{\alpha}$ phase, the hydrocarbon chains are disordered (and the phase is liquid-like), while they order by stretching and tilting in the $L_{\beta^{\prime}}$ (solid-like) phase. Several theoretical models have been proposed in the past to describe the main transition in isolated membranes [21,22].

In other single-component lipid systems, however, a rippled $\left(P_{\beta^{\prime}}\right)$ phase appears between the $L_{\alpha}$ and $L_{\beta^{\prime}}$ phases for stacked bilayer membranes $23,24,25]$. This phase is more peculiar and many studies have been devoted to understand better its modulation wavelength and amplitude 26. For a stack of lipid bilayers in water (a lamellar phase), the phase diagram as a function of relative humidity and temperature was reported [27,28]. Several attempts have been made to describe the rippled phase theoretically such as those based on a molecular level description [29, 30], or Monte Carlo simulations [31]. In continuum theories of the rippled phase, different quantities have been suggested for the order parameter. Examples are the membrane thickness [19,32] or the configuration of the hydrocarbon chains (ratio of the trans bonds in the chains) [33. In both cases the order parameter is scalar. More recently, a Ginzburg-Landau theory was proposed by Chen, Lubensky, and MacKintosh (CLM) who employed a vectorial order parameter representing the tilt of the lipid molecules [34,35].

We further discuss the CLM model in Sec. 4 below, but our own starting point is based on a model proposed by Goldstein and Leibler (GL) for single-component lipid membranes [19,20]. To describe the main transition involving chain ordering and stiffening, GL introduced a dimensionless scalar order parameter of the membrane, $m(\mathbf{r})$ :

$$
m(\mathbf{r})=\frac{\delta(\mathbf{r})-\delta_{0}}{\delta_{0}}
$$

This parameter depends on the actual membrane thickness $\delta(\mathbf{r})$, and on the constant membrane thickness $\delta_{0}$ of the $L_{\alpha}$ phase. The two-dimensional lateral position within the bilayer plane is denoted by $\mathbf{r}$. Notice that $m$ encapsulates changes that may occur in several degrees of freedom, including, for example, the conformations of the hydrocarbon chains, molecular tilt and positional ordering. In the present paper, the bilayer nature of the membrane is not taken into account and the bilayer thickness is simply taken as a sum of the two monolayer thicknesses. The role of the bilayer structure in the formation of rippled phases was explicitly considered in previous models [36,37].

For simplicity, we assume that the rippled phase is spatially modulated only in the $x$-direction, and ignore two-dimensional rippled phases such as the square lattice phase [34,35] or the hexagonal phase [38,39]. For an isolated lipid bilayer membrane, the stretching free energy per lipid molecule is [19]

$$
\begin{aligned}
f_{\mathrm{st}} & =\frac{1}{2} a_{2} m^{2}+\frac{1}{3} a_{3} m^{3}+\frac{1}{4} a_{4} m^{4} \\
& +\frac{1}{2} C\left(\frac{\mathrm{d} m}{\mathrm{~d} x}\right)^{2}+\frac{1}{2} D\left(\frac{\mathrm{d}^{2} m}{\mathrm{~d} x^{2}}\right)^{2} .
\end{aligned}
$$

A very similar free energy was proposed by Marder et al. [32. The first three terms are the Landau expansion in powers of the order parameter $m$. Only $a_{2}$, the second order term coefficient, has an explicit temperature dependence: $a_{2}=a_{2}^{\prime}\left(T-T^{*}\right)$, with $T^{*}$ being a reference temperature. (It actually is the critical temperature in the absence of the cubic term.) Because the $L_{\beta^{\prime}} \rightarrow L_{\alpha}$ phase transition is known to be first-order, $a_{3}$ is taken to be negative, whereas $a_{4}$ is always positive to ensure stability of this free energy expansion. The next two terms are related to the lowest order gradients of $m$. (Note that the $x$-coordinate is rescaled in units of a molecular length scale $\ell$ so that $x \rightarrow x / \ell$ is dimensionless.) These gradient terms represent elastic out-of-plane undulations of the membrane and their coefficients are the elastic constants $C$ and $D$, respectively. The coefficient $C$ can be either positive or negative, but $D$ is always positive in order to ensure stability of the expansion. The physical origin of a negative $C$ value can be related to the coupling between the conformation of the chains and the curvature of the lipid/water interface, or to interactions between the polar head-groups of the lipids and water [19]. This point will be further discussed later in Sec. 4. In the absence of spatial gradient terms in Eq. (2) and if $m(x)$ is constant in space, the main transition temperature $T_{\mathrm{m}}$ is calculated from the conditions $f_{\mathrm{st}}=\mathrm{d} f_{\mathrm{st}} / \mathrm{d} m=0$. It is related to $T^{*}$ by

$$
T_{\mathrm{m}}=T^{*}+\frac{2 a_{3}^{2}}{9 a_{2}^{\prime} a_{4}} .
$$

With nonzero $a_{3}$, a first-order melting transition at $T_{\mathrm{m}}$ preempts the critical point at $T^{*}$. 


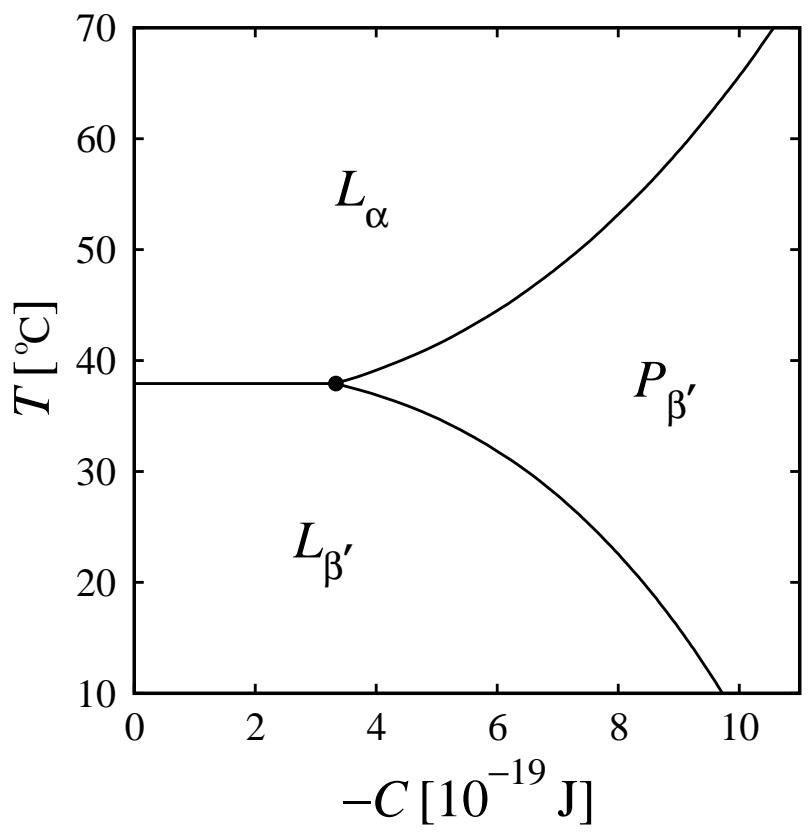

Fig. 1. Mean-field phase diagram of a single-component lipid membrane as a function of (negative) elastic constant $C$ and temperature $T$ (in degrees Celsius). The liquid $\left(L_{\alpha}\right)$, solid $\left(L_{\beta^{\prime}}\right)$ and rippled $\left(P_{\beta^{\prime}}\right)$ phases are separated from one another by first-order phase transition lines which meet at a triple point (marked by a full circle): $C_{\mathrm{tr}}=-3.33 \times 10^{-19} \mathrm{~J}, T_{\mathrm{tr}}=38^{\circ} \mathrm{C}$. The parameters used to calculate this phase diagram are given in the text.

When $C>0$, the equilibrium phase is always homogeneous in space; either as a $L_{\beta^{\prime}}$ or $L_{\alpha}$ phase. On the other hand, for $C<0$, the rippled phase with a characteristic wave vector $q^{*}=(-C / 2 D)^{1 / 2}$ (in unit of $\ell^{-1}$ ) may dominate over the two other phases. (It should be noted that $C<0$ is only a necessary condition to have the rippled phase as will be discussed below.) To obtain the mean-field phase diagram, we employ the single-mode approximation in which the rippled phase is described by the most dominant wavevector $q^{*}$, only. This approximation is valid near the phase transition point where the segregation tendency between the two lipids is weak enough. The order parameter $m$ can be written as 32

$$
m=m_{0}+m_{1} \cos \left(q^{*} x\right),
$$

where $m_{0}=\langle m\rangle$ is the spatial average of $m$, and $m_{1}$ is the amplitude of the single $q^{*}$-mode of the rippled phase taken to lie arbitrarily in the $x$-direction.

By substituting Eq. (4) into Eq. (2) and taking the spatial average over one period, we obtain

$$
\begin{aligned}
\left\langle f_{\mathrm{st}}\right\rangle= & -\frac{C^{2}}{16 D} m_{1}^{2}+\frac{1}{2} a_{2}\left(m_{0}^{2}+\frac{1}{2} m_{1}^{2}\right) \\
& +\frac{1}{3} a_{3}\left(m_{0}^{3}+\frac{3}{2} m_{0} m_{1}^{2}\right) \\
& +\frac{1}{4} a_{4}\left(m_{0}^{4}+3 m_{0}^{2} m_{1}^{2}+\frac{3}{8} m_{1}^{4}\right) .
\end{aligned}
$$

The two homogeneous liquid and solid phases, $L_{\alpha}$ and $L_{\beta^{\prime}}$, respectively, are characterized by $m_{1}=0$; while $m_{0}=0$ in the disordered liquid $L_{\alpha}$ phase, and is non-zero in the solid $L_{\beta^{\prime}}$ phase. From the three phases only the $P_{\beta^{\prime}}$ phase is characterized by a modulating amplitude, $m_{1} \neq 0$.

The mean-field phase diagram is obtained by minimizing Eq. (5) with respect to both $m_{0}$ and $m_{1}$, and comparing the relative stability of the three relevant phases. In Fig. 1, we present an example of such a phase diagram, as function of the (negative) effective elastic constant $C$ and temperature $T$ plotted in degrees Celsius. The parameter $C$ can be a function of relative humidity (fraction of water content in the lamellar phase) as discussed below. The phase diagram is calculated for a choice of system parameters which reproduce the main transition temperature of DMPC at $T_{\mathrm{m}}=38^{\circ} \mathrm{C}: a_{2}^{\prime}=2.4 \times 10^{-21} \mathrm{~J} \mathrm{~K}^{-1}$, $a_{3}=-1.1 \times 10^{-18} \mathrm{~J}, a_{4}=2.2 \times 10^{-18} \mathrm{~J}, T^{*}=-13^{\circ} \mathrm{C}$ $(260 \mathrm{~K})$, and $D=2.0 \times 10^{-18} \mathrm{~J}$. These values (except $D>0$ whose value is less important) are taken from Ref. 20]. By inserting the above Landau coefficients into Eq. (3), it can be readily checked that the main transition temperature of DMPC $T_{\mathrm{m}}=38^{\circ} \mathrm{C}$ (Fig. 11) is obtained.

In Fig. 1, three first-order phase transition lines separate the three phases and meet at a triple point, $C_{\mathrm{tr}}=$ $-3.33 \times 10^{-19} \mathrm{~J}$. Due to the first-order nature of the $L_{\beta^{\prime}} \rightarrow$ $L_{\alpha}$ phase transition, it can be argued, on general grounds, that the triple point $C_{\mathrm{tr}}$ has to be located at non-zero values of $C$. For small magnitude of $C,|C|<\left|C_{\mathrm{tr}}\right|$, the horizontal first-order phase transition line occurs at $T=$ $T_{\mathrm{m}}=38^{\circ} \mathrm{C}$ and is $C$ independent. An increase in temperature (as long as $|C|<\left|C_{\text {tr }}\right|$ ) will melt the solid phase $L_{\beta^{\prime}}$ directly into the liquid phase $L_{\alpha}$ at $T_{\mathrm{m}}$. But for negative and large enough magnitude of $C,|C|>\left|C_{\mathrm{tr}}\right|$, any increase in temperature will cause a sequence of phase transitions: first the solid phase $L_{\beta^{\prime}}$ melts into the $P_{\beta^{\prime}}$ phase, and only then, upon further increase of the temperature, the $P_{\beta^{\prime}}$ phase will make a phase transition into the liquid phase $L_{\alpha}$. It is also apparent from the figure that the region of the $P_{\beta^{\prime}}$ phase expands on the expense of the uniform liquid and solid phases as $|C|$ increases. This means that the phase transitions $L_{\beta^{\prime}} \rightarrow P_{\beta^{\prime}}$ and $P_{\beta^{\prime}} \rightarrow L_{\alpha}$ (at constant $C$ ) occur at larger temperature deviations from $T_{\mathrm{m}}$ as $|C|$ increases.

The phase diagram of Fig. 1 essentially reproduces all the experimental facts observed for single-component lipid membranes. It qualitatively agrees with the observed phase diagram of a lamellar phase of DMPC, when the negative elastic constant $C$ is taken to be proportional to the relative humidity [27,28]. Although the exact dependence of the elastic constant $C$ on humidity is not known, the former can possibly be reduced by increasing hydration. For another lipid, DPPC, the $P_{\beta^{\prime}}$ phase is found experimentally to occur at temperatures around $37^{\circ} \mathrm{C}$ (in Ref. 18 the precise temperatures for the $L_{\beta^{\prime}} \rightarrow P_{\beta^{\prime}}$ and $P_{\beta^{\prime}} \rightarrow L_{\alpha}$ phase transitions have not been fully reported), while the rippled phase is not observed for a stack DPPE lipid bilayers. Such a difference in the lipid phase behavior can be qualitatively attributed to different values of the elastic constant $C$. 


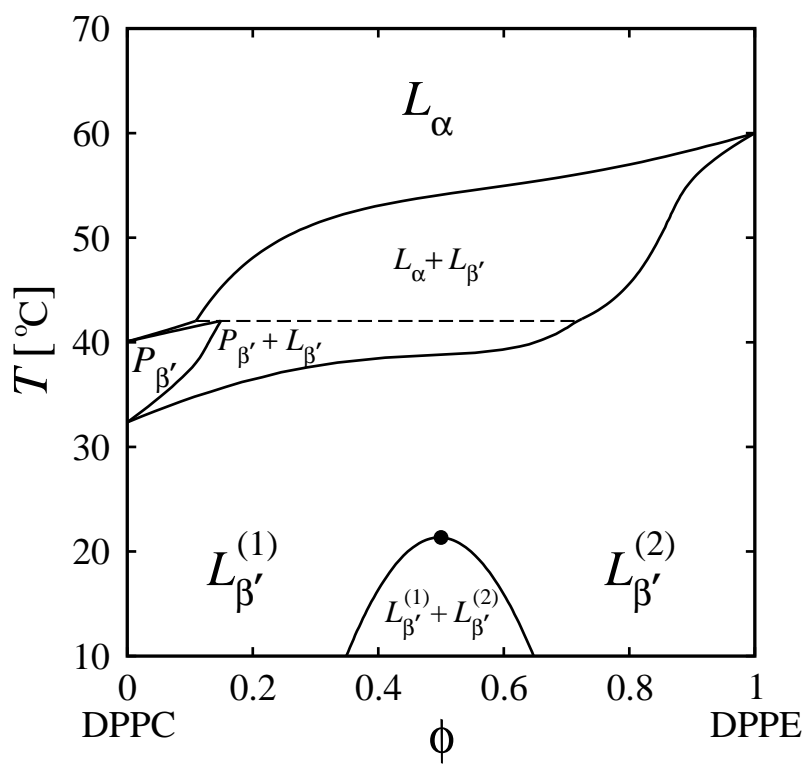

Fig. 2. Calculated mean-field phase diagram of a binary lipid mixture as a function of their relative composition $\phi$ and temperature $T$. The parameter values are chosen to fit DPPC/DPPE mixtures $(\phi=0$ represents pure DPPC and is denoted by a subscript "B"): $C_{\mathrm{A}}=-2.0 \times 10^{-19} \mathrm{~J}, C_{\mathrm{B}}=-5.2 \times$ $10^{-19} \mathrm{~J}, T_{\mathrm{A}}^{*}=9^{\circ} \mathrm{C}, T_{\mathrm{B}}^{*}=-15^{\circ} \mathrm{C}$, and $J=1.45 \times 10^{-20} \mathrm{~J}$. All other parameter values and definitions of the different phases are the same as in Fig. 1 The critical point is indicated by a full circle and occurs at $T_{\mathrm{c}}=21.3^{\circ} \mathrm{C}$. The horizontal dashed line indicates the three-phase coexistence at the triple point, $T_{\operatorname{tr}}=42.0^{\circ} \mathrm{C}$.

\section{Two-component lipid mixtures}

In this section, we extend the above single-component model to membranes consisting of a binary lipid mixture. We employ a similar approach as was used in our previous study [15, 16], and consider the coupling of the melting phase transition with a lateral phase separation in the mixed membrane.

A binary mixed membrane is modeled as an incompressible A/B mixture of $\phi$ mole fraction of lipid A and $(1-\phi)$ fraction of lipid B. For simplicity, we assume the same area per molecule for both species and ignore any lipid exchange with the surrounding solvent. In general, the two lipids will have different main transition temperatures originating from different molecular parameters such as chain length, degree of saturation and hydrophilic head group.

The total free energy per lipid, $f_{\text {tot }}=f_{\text {st }}+f_{\text {mix }}$, comprises: (i) the chain stretching free energy $f_{\text {st }}$ given by Eq. (2), and (ii) the free energy of mixing, $f_{\text {mix }}$. The latter energy per lipid molecule is the sum of the entropy of mixing and enthalpy. It can be written within the BraggWilliams (mean-field) approximation as

$$
f_{\text {mix }}=k_{\mathrm{B}} T[\phi \log \phi+(1-\phi) \log (1-\phi)]+\frac{1}{2} J \phi(1-\phi),
$$

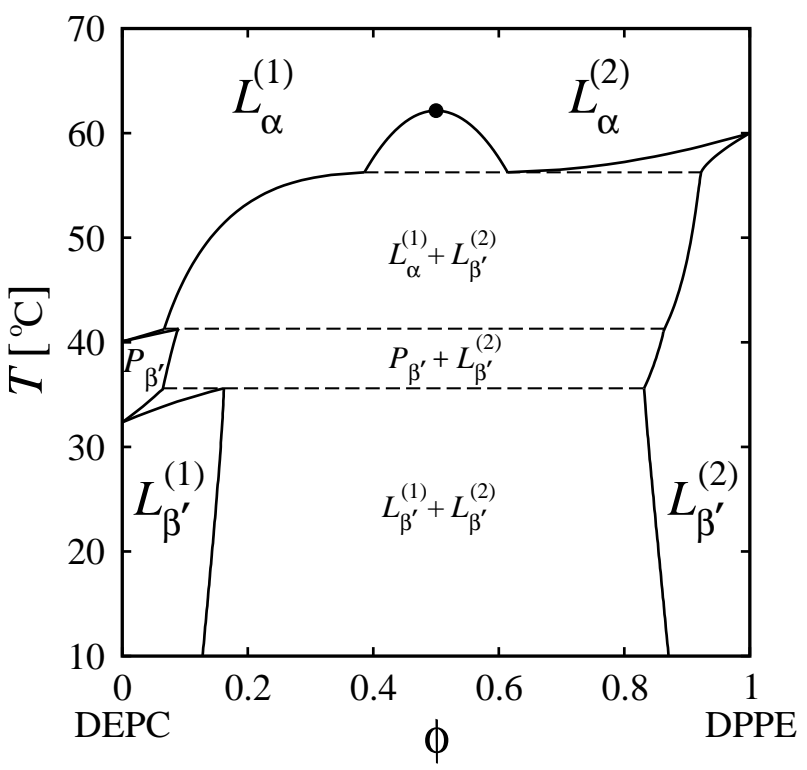

Fig. 3. Calculated mean-field phase diagram of a binary lipid mixture as a function of their relative composition $\phi$ and temperature $T$. The parameters are the same as in Fig. 2] except $J=1.85 \times 10^{-20} \mathrm{~J}$. The critical point is located at $62.1^{\circ} \mathrm{C}$, and three triple points occur at $35.6,41.3$, and $56.3^{\circ} \mathrm{C}$, respectively. This choice of model parameters is in agreement with experimental phase diagram of DEPC/DPPE mixture where $\phi=0$ represents pure DEPC.

where $k_{\mathrm{B}}$ is the Boltzmann constant, and $J>0$ is an attractive interaction parameter between the lipids that enhances lipid-lipid demixing.

For a binary mixture the free energy $f_{\text {st }}$ is assumed to have the same functional dependence on the effective elastic constant $C$ and the reference temperature $T^{*}$. Although these two parameters depend on the lipid composition $\phi$, the precise dependence cannot be calculated from such a phenomenological approach. Alternatively, we proceed by further assuming the simplest linear interpolation between the two pure lipid limiting values:

$$
\begin{aligned}
& C(\phi)=\phi C_{\mathrm{A}}+(1-\phi) C_{\mathrm{B}}, \\
& T^{*}(\phi)=\phi T_{\mathrm{A}}^{*}+(1-\phi) T_{\mathrm{B}}^{*},
\end{aligned}
$$

where $C_{\mathrm{A}}$ and $C_{\mathrm{B}}$ are the elastic constants, and $T_{\mathrm{A}}^{*}$ and $T_{\mathrm{B}}^{*}$ are the reference temperatures of lipid $\mathrm{A}$ and $\mathrm{B}$, respectively. The other elastic constant $D$, as well as $a_{3}$ and $a_{4}$ in Eq. (2) are assumed to be the same for the two lipids because the important properties of the lipids are mostly reflected in the choice of their $C$ and $T^{*}$ parameter values. As for all other model parameters, we use hereafter the same values listed in the previous section.

The total free energy $f_{\text {tot }}=f_{\text {st }}+f_{\text {mix }}$ as function of $m$ and $\phi$ is now readily available by substituting Eqs. (7) and (8) into Eq. (2). There is an explicit coupling between the two order parameters, $m$ and $\phi$ originating from the free energy $f_{\text {st }}=f_{\text {st }}(m, \phi)$. Note that the first term in Eq. (2) and Eq. (8) lead to a coupling term which scales 
as $\phi m^{2}$. Another possible coupling term is $\phi^{2} m$ that simply renormalizes the interaction parameter $J$ of $f_{\text {mix }}$ and is less interesting. The two-phase coexistence region in the $(T, \phi)$ plane is calculated by using the common tangent construction to account for the constraint value of the relative concentration $\phi$, after minimizing $f_{\text {tot }}$ with respect to $m_{0}$ and $m_{1}$.

By properly choosing the model parameters, we attempt to reproduce the binary phase diagram of several lipid mixtures, the first being that of DPPC/DPPE [18] shown in Fig. 2. Since a pure DPPE membrane does not show experimentally the rippled phase, its elastic constant is chosen as $C_{\mathrm{A}}=-2.0 \times 10^{-19} \mathrm{~J}$. Namely, below the triple point $C_{\mathrm{tr}}$ (Fig. (1). The DPPE reference temperature $T_{\mathrm{A}}^{*}$ is calculated from Eq. (3) with the same values for the Landau coefficients as before, by fitting its main transition temperature to the observed one of $T_{\mathrm{m}}=60^{\circ} \mathrm{C}$. On the other hand, the elastic constant of DPPC is chosen as $C_{\mathrm{B}}=-5.2 \times 10^{-19} \mathrm{~J}$ (above $C_{\mathrm{tr}}$ ) so that the $P_{\beta^{\prime}}$ phase appears between the $L_{\beta^{\prime}}$ and $L_{\alpha}$ phases. The reference temperature of DPPC is set as $T_{\mathrm{B}}^{*}=-15^{\circ} \mathrm{C}$, corresponding, from Eq. (3), to a $L_{\beta^{\prime}} \rightarrow L_{\alpha}$ transition temperature $T_{\mathrm{m}}=36^{\circ} \mathrm{C}$. This temperature is not available from experiments but lies in-between the experimentally observed $L_{\beta^{\prime}} \rightarrow P_{\beta^{\prime}}$ phase transition temperature at $32^{\circ} \mathrm{C}$ and the $P_{\beta^{\prime}} \rightarrow L_{\alpha}$ one at $40^{\circ} \mathrm{C}$.

All the above chosen parameter values are used to calculate the binary phase diagram fitting the DPPC/DPPE mixture. The relative composition $\phi$ is chosen such that $\phi=0$ corresponds to pure DPPC, while $\phi=1$ to pure DPPE. In addition, the interaction parameter is chosen as $J=1.45 \times 10^{-20} \mathrm{~J}$. When the temperature is relatively low, there is a coexistence region between two $L_{\beta^{\prime}}$ phases (denoted by $L_{\beta^{\prime}}^{(1)}+L_{\beta^{\prime}}^{(2)}$ ). This low temperature coexistence terminates at a critical temperature, above which there is only one $L_{\beta^{\prime}}$ phase for the entire range of composition $\phi$. It should be noted, however, that the appearance of the critical point between the two "solid" phases is an artifact of the model. As the temperature is further increased, the $P_{\beta^{\prime}}$ phase appears close to the pure DPPC axis. It is bound by a coexistence region with the $L_{\beta^{\prime}}$ phases as well as with another (much smaller in its extent) coexistence region with the $L_{\alpha}$ phase. This latter region is very small on the scale of the figure and collapses almost into a line. These two coexistence regions terminate at a triple point temperature, $T=T_{\mathrm{tr}}$, at which all the three phases coexist. For even higher temperature, $T>T_{\mathrm{tr}}$, the rippled phase disappears and the only coexistence region is between the $L_{\alpha}$ and $L_{\beta^{\prime}}$ phases.

The calculated phase diagram is in quantitative agreement with the experimental one for DPPC/DPPE mixtures (e.g., Fig. 7 of Ref. [18]). Even the coexistence between the two solid phases is alluded in Ref. [18]. A similar type of phase diagram was obtained for mixtures of DMPC/DPPS and DPPC/DPPS [17. In these lipid mixtures, both DMPC and DPPC exhibit the rippled phase, while pure DPPS undergoes a direct transition from the $L_{\beta^{\prime}}$ to $L_{\alpha}$ phases similar to DPPE.

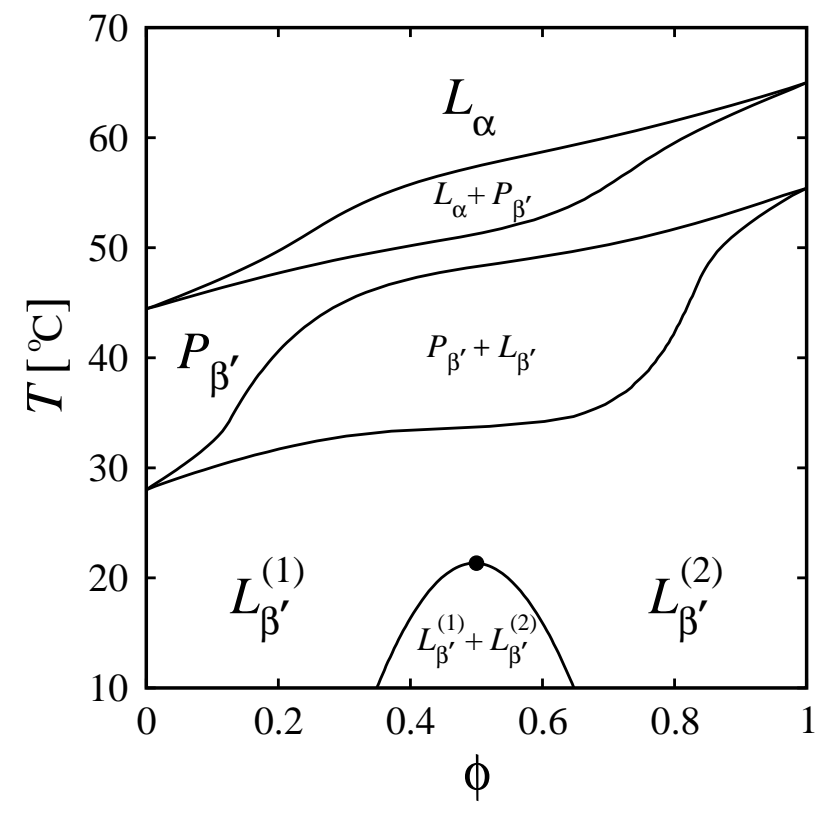

Fig. 4. Mean-field phase diagram of a binary mixture of two lipids as a function of the relative composition $\phi$ and temperature $T$. All parameters are the same as in Fig. 2 except $C_{\mathrm{A}}=-5.5 \times 10^{-19} \mathrm{~J}$ and $C_{\mathrm{B}}=-6.5 \times 10^{-19} \mathrm{~J}$. The lipid interaction parameter is set to be $J=1.45 \times 10^{-20} \mathrm{~J}$. The critical point is located at $21.3^{\circ} \mathrm{C}$ as in Fig. 2 ,

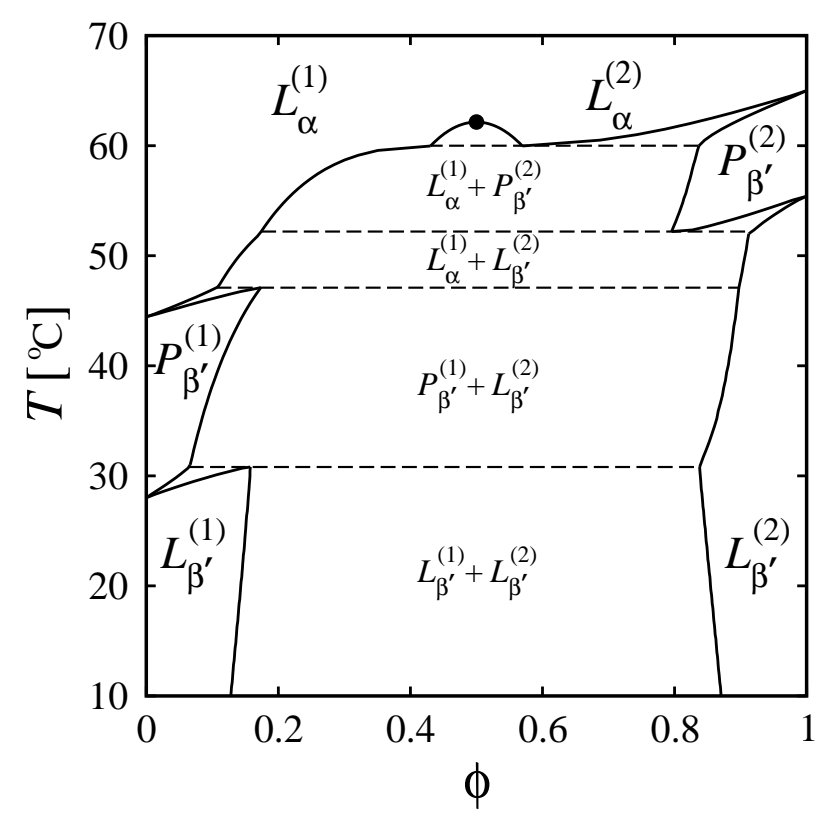

Fig. 5. Mean-field phase diagram of a binary mixture of two lipids as a function of the relative composition $\phi$ and temperature $T$. All parameters are the same as in Fig. 4 except $J=1.85 \times 10^{-20} \mathrm{~J}$. The critical point is located at $62.1^{\circ} \mathrm{C}$ as in Fig. 3. and four triple points occur at 30.8, 47.1, 52.2, and $60.0^{\circ} \mathrm{C}$, respectively. 
An even stronger lipid-lipid segregation can be modeled by increasing the value of the lipid interaction parameter to $J=1.85 \times 10^{-20} \mathrm{~J}$, while keeping all other parameter values unchanged. In the resulting phase diagram shown in Fig. 3, the lower coexistence region between the two $L_{\beta^{\prime}}$ phases penetrates the other coexistence regions. Such a phase diagram resembles that of DEPC/DPPE lipid mixtures 13. This combination of lipids have a strong segregation tendency because both head and tail moieties are different. In the calculated phase diagram, there are three triple points indicated by the horizontal dashed lines. The rippled phase can now coexist with either of the $L_{\beta^{\prime}}$ (regions $P_{\beta^{\prime}}+L_{\beta^{\prime}}^{(1)}$ or $P_{\beta^{\prime}}+L_{\beta^{\prime}}^{(2)}$ in the figure) as well as with the $L_{\alpha}$ phase $\left(P_{\beta^{\prime}}+L_{\alpha}^{(1)}\right)$. There is also a two-phase coexistence region between the two liquid phases $\left(L_{\alpha}^{(1)}+L_{\alpha}^{(2)}\right)$. The three-phase coexistence between the two $L_{\alpha}$ phases and the $L_{\beta^{\prime}}$, and the existence of the critical point have been indeed observed for DEPC/DPPE lipid mixtures [13].

In Figs. 4 and 5 we calculate the phase diagrams in situations where each of the two pure lipids exhibit a rippled phase between their corresponding $L_{\alpha}$ and $L_{\beta^{\prime}}$ phases. Hence, we choose the magnitudes of the elastic constant to be larger than $\left|C_{\mathrm{tr}}\right|$ of Fig. 1. $C_{\mathrm{A}}=-5.5 \times 10^{-19} \mathrm{~J}$ and $C_{\mathrm{B}}=-6.5 \times 10^{-19} \mathrm{~J}$. The choices of the reference temperatures $T_{\mathrm{A}}^{*}$ and $T_{\mathrm{B}}^{*}$, as well as all other parameters, is the same as in Figs. 2 and 3. The interaction parameter is taken as $J=1.45 \times 10^{-20} \mathrm{~J}$ in Fig. 4, and have a some-

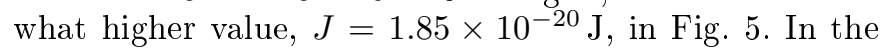
phase diagram of Fig. 4, one region of the rippled phase extends throughout the entire range of the lipid composition. Above and below this region, the rippled phase coexists with the $L_{\beta^{\prime}}$ and $L_{\alpha}$ phases. At low temperatures, another coexistence region can be seen between the two $L_{\beta^{\prime}}$ phases. For a larger interaction parameter $J$ (Fig. (5), these coexistence regions merge to form a large two-phase region, and there are four triple points. Here we see a new coexistence between $L_{\alpha}$ and $L_{\beta^{\prime}}^{(2)}$ phases which does not exist in Fig. 4. Although such more complex phase diagrams have not been yet reported in experiments, we expect that they can be found in the future by properly choosing the lipid mixtures. One possible choice of lipids that may give rise to such a phase behavior is the DPPC/DMPC mixture. Both lipids exhibit the rippled phase between their $L_{\beta^{\prime}}$ and $L_{\alpha}$ phases.

\section{Summary and discussion}

In this paper we proposed a model describing the liquidsolid coexistence region in membranes composed of a binary lipid mixture. We addressed in particular the possible existence of a rippled phase between the liquid and solid ones, and its effect on the global phase diagrams. In order to incorporate the possibility of a rippled phase, we use a model previously proposed for single-component membranes by Goldstein and Leibler (GL) [19,20]. Their main purpose was to present a model describing the lyotropic lamellar phases of lipid bilayers as stacks of interacting membranes. Here we have shown explicitly that the GL lipid stretching energy of an isolated membrane exhibits the sequential phase transitions $L_{\beta^{\prime}} \rightarrow P_{\beta^{\prime}} \rightarrow L_{\alpha}$ by increasing the temperature. We further extend the singlecomponent model and apply it to binary lipid mixtures assuming that the elastic parameter $C$ as well as the reference temperature $T^{*}$ depend linearly on the relative composition of the two lipids. The calculated phase diagrams are in quantitative agreement with the experimental ones for specific lipid mixtures. We have also predicted other types of phase diagrams, which have yet to be checked experimentally for other lipid mixtures.

Several points merit further discussion. In Sec. 2, we choose the relative membrane thickness as the scalar order parameter to describe the membrane phase transitions [19. 20. In another model proposed by Chen, Lubensky and MacKintosh (CLM), a two-dimensional molecular tilt was used as a vector order parameter [34,35]. In their model, a coupling mechanism between the membrane curvature and the gradient in molecular tilt was considered. As a result, various types of rippled phases with different inplane symmetries have been predicted.

In spite of the success in predicting, e.g., the square lattice phase that was found in the experiments [40], the CLM model suffers from some deficiencies which makes it inappropriate to be used in our study. Within the CLM model, the $L_{\beta^{\prime}} \rightarrow P_{\beta^{\prime}}$ phase transition cannot be induced only by changing the temperature, while keeping all other system parameters fixed. In other words, the phase boundaries in the CLM model is parallel to the temperature axis (see, for example, Fig. 9 in Ref. [35]). This is essentially a result of the vector nature of the CLM order parameter 411. Unfortunately, such a phase behavior is not in accord with the experimental phase diagrams [27,28], where the $L_{\beta^{\prime}}-P_{\beta^{\prime}}$ phase boundary depends on temperature. This is the main reason why we have chosen to use a scalar order parameter giving us the phase diagram as in Fig. 1 .

The second problem is that the CLM model predicts a second-order phase transition between the $L_{\alpha}$ and $L_{\beta^{\prime}}$ phases. However, the main transition is known to be firstorder according to various experimental investigations [19, 20], as was previously mentioned in Ref. [40. A possible way out would be to include the 6 th order term of the tilting vector in the CLM Landau expansion, this lies beyond the scope of the present work.

The two problems mentioned above do not exist in the GL model. However, since the choice of a single scalar as the order parameter is a major simplification, many degrees of freedom of the lipid molecules are not properly taken into account. For example, one cannot distinguish between the similar $L_{\beta^{\prime}}$ and $L_{\beta}$ phases because the scalar order parameter $m$ cannot model the molecular tilt characteristic of the solid-like $L_{\beta}$ phase. With this choice of order parameter, one can only distinguish between a dilute and a condensed phase. Because the membrane thickness does not account for the different symmetries of the $L_{\alpha}$, $L_{\beta^{\prime}}$, and $P_{\beta^{\prime}}$ phases, $m$ cannot represent any crystalline order, and the solid phase is not properly described here. Hence the appearance of the critical points between the 
two $L_{\beta^{\prime}}$ phases in Figs. 2 and 4 is an artifact of our model. Although this problem is remedied in the CLM model, the GL model captures the essential features of the structural transitions in lipid membranes in the presence of the rippled phase. Using the latter model is sufficient to reproduce even quantitatively some of the global features of phase diagrams of binary lipid mixtures, in agreement with experiments.

In Eq. (2) we assumed for simplicity that the rippled phase is modulated spatially only in the $x$-direction. In general, one can consider the full $(x, y)$ inplane modulations as treated in the CLM model [34,35]. This can be accomplished by including in Eq. (2) additional gradient terms in the $y$-direction. The resulting phase diagram will be more complex because there can be more than one rippled phase. Although a two-dimensional square lattice phase has been identified for a single-component DTPC lipid [40, it has not yet been found in binary lipid mixtures. We also note that the one-dimensional modulation of the order parameter expressed by Eq. (4) is similar to the $P_{\beta^{\prime}}^{(2)}$ phase in CLM, with the main difference that we include a non-zero average term $m_{0}$. The physical interpretation of the actual order parameter in the two models differs as is explained above.

Finally, we discuss why the elastic coefficient $C$ can take negative values in Eq. (2) as is required to get the $P_{\beta^{\prime}}$ phase at equilibrium. In Sec. 2, we have mentioned that the coupling between the conformation of the chains and the curvature of the membrane may be a possible reason [19. In general, if there exists a coupling between an additional elastic degree of freedom and the gradient of the order parameter, the elastic constant $C$ will be reduced. This situation can be expressed by including the following two additional terms in the free energy:

$$
f_{\mathrm{el}}=\frac{1}{2} B \rho^{2}-\gamma \rho\left(\frac{\mathrm{d} m}{\mathrm{~d} x}\right)
$$

Here $\rho$ represents some elastic degree of freedom, $B$ is another positive elastic constant, and $\gamma$ is a coupling constant. For example, $\rho$ can be taken as the curvature of the lipid/water interface, and $B$ is the bending rigidity of the membrane. In the second term, $\rho$ is linearly coupled to the gradient of $m$ which induces a spontaneous value of $\rho$. Minimizing $f_{\text {el }}$ of Eq. (9) with respect to $\rho$, we get

$$
\rho=\frac{\gamma}{B}\left(\frac{\mathrm{d} m}{\mathrm{~d} x}\right) .
$$

This means that the equilibrium value of $\rho$ is proportional to the gradient of $m$. By substituting this equation back into Eq. (9), the effective elastic constant becomes

$$
C \rightarrow C-\gamma^{2} / B
$$

in Eq. (2). Hence $C$ is reduced by $\gamma^{2} / B$ and can reach negative values when the coupling constant $\gamma$ is large enough (or when the elastic constant $B$ is small enough). Although it goes beyond the scope of the present paper to suggest specific molecular mechanism to account for this added elastic degree of freedom, such a mechanism would, in principle, explain the negative value of the effective elastic constant, $C$.

This work is supported by KAKENHI (Grant-in-Aid for Scientific Research) on Priority Areas "Soft Matter Physics" and Grant no. 18540410 from the Ministry of Education, Culture, Sports, Science and Technology of Japan. One of us (DA) acknowledges support from the Israel Science Foundation (ISF) under grant no. 160/05 and the US-Israel Binational Foundation (BSF) under grant no. 287/02

\section{References}

1. K. Simons and E. Ikonen, Nature 387, 569 (1997).

2. K. Simons and E. Ikonen, Science 290, 1721 (2000).

3. D. M. Engelman, Nature 438, 578 (2005).

4. A. Leibler and D. Andelman, J. Phys. (Paris) 48, 2013 (1987).

5. S. L. Veatch and S. L. Keller, Phys. Rev. Lett. 89, 268101 (2002).

6. T. Baumgart, S. T. Hess, and W. W. Webb, Nature 425, 821 (2003).

7. S. L. Veatch and S. L. Keller, Phys. Rev. Lett. 94, 148101 (2005).

8. S. L. Veatch and S. L. Keller, Biochim. Biophys. Acta 1746, $172(2005)$.

9. E. London, Biochim. Biophys. Acta 1746, 203 (2005).

10. L. A. Bagatolli and E. Gratton, Biophys. J. 78, 290 (2000).

11. P. A. Beales, V. D. Gordon, Z. Zhao, S. U. Egelhaaf, and W. C. K. Poon, J. Phys.: Cond. Matt. 17, S3341 (2005).

12. S. Schneider and G. Gompper, Europhys. Lett. 70, 136 (2005).

13. S. H. Wu and H. M. McConnell, Biochemistry 14, 847 (1975).

14. K. Arnold, A. Lösche, and K. Gawrisch, Biochim. Biophys. Acta 645, 143 (1981).

15. S. Komura, H. Shirotori, P. D. Olmsted, and D. Andelman, Europhys. Lett. 67, 321 (2004).

16. S. Komura, H. Shirotori, and P. D. Olmsted, J. Phys.: Condens. Matter 17, S2951 (2005).

17. E. J. Luna and H. M. McConnell, Biochim. Biophys. Acta 470, 303 (1977).

18. A. Blume, R. J. Wittebort, S. K. Das Gupta, and R. G. Griffin, Biochemistry 21, 6243 (1982).

19. R. E. Goldstein and S. Leibler, Phys. Rev. Lett. 61, 2213 (1988).

20. R. E. Goldstein and S. Leibler, Phys. Rev. A 40, 1025 (1989).

21. S. Doniach, J. Chem. Phys. 68, 4912 (1978).

22. T. Izuyama and Y. Akutsu, J. Phys. Soc. Jpn. 51, 730 (1982).

23. J. A. N. Zasadzinski, J. Schneir, J. Gurley, V. Elings, and P. K. Hansma, Science 239, 1013 (1988).

24. D. C. Wack and W. W. Webb, Phys. Rev. Lett. 61, 1210 (1988).

25. M. P. Hentschel and F. Rustichelli, Phys. Rev. Lett. 66, 903 (1991).

26. B. A. Cunningham, A.-D. Brown, D. H. Wolfe, W. P. Williams, and A. Brain, Phys. Rev. E 58, 3662 (1998). 
27. E. B. Sirota, G. S. Smith, C. R. Safinya, R. J. Plano, and N. A. Clark, Science 242, 1406 (1988).

28. G. S. Smith, E. B. Sirota, C. R. Safinya, and N. A. Clark, Phys. Rev. Lett. 60, 813 (1988).

29. S. Doniach, J. Chem. Phys. 70, 4587 (1979).

30. J. M. Carlson and J. P. Sethna, Phys. Rev. A 36, 3359 (1987).

31. W. S. McCullough and H. L. Scott, Phys. Rev. Lett. 65, 931 (1990).

32. M. Marder, H. L. Frisch, J. S. Langer, and H. M. McConnell, Proc. Natl. Acad. Sci. USA 81, 6559 (1984).

33. K. Honda and H. Kimura, J. Phys. Soc. Jpn. 60, 1212 (1991).

34. T. C. Lubensky and F. C. MacKintosh, Phys. Rev. Lett. 71, 1565 (1993).

35. C.-M. Chen, T. C. Lubensky, and F. C. MacKintosh, Phys. Rev. E 51, 504 (1995).

36. H. Kodama and S. Komura, J. Phys. II (France) 3, 1305 (1993).

37. U. Seifert, J. Shillcock, and P. Nelson, Phys. Rev. Lett. 77, 5237 (1996).

38. C.-M. Chen and F. C. MacKintosh, Europhys. Lett. 30, 215 (1995).

39. C.-M. Chen and F. C. MacKintosh, Phys. Rev. E 53, 4933 (1996).

40. L. Yang and M. Fukuto, Phys. Rev. E 72, 010901 (2005).

41. P. Chaikin and T. C. Lubensky, Principles of condensed matter physics (Cambridge Univ., Cambridge, 1995). 